\title{
Computer-assisted cognitive remediation in adolescents with psychosis or at risk for psychosis: a 6-month follow-up
}

Urben S, Pihet S, Jaugey L, Halfon O, Holzer L. Computer-assisted cognitive remediation in adolescents with psychosis or at risk for psychosis: a 6-month follow-up.

Objective: To investigate short-term outcomes of a computer-assisted cognitive remediation (CACR) for adolescents with psychotic disorders or at high risk for psychosis.

Method: Cognitive abilities and clinical status were assessed at baseline $(N=32)$ and at 6-month follow-up $(N=22)$ after enrolment in either a CACR (treatment group) or a computer games (control group) program (8 weeks).

Results: With regard to the cognitive abilities, no amelioration was found in the control group while, in the CACR group, significant improvements in inhibition $(p=0.040)$ and reasoning $(p=0.005)$ were observed. Furthermore, symptom severity decreased significantly in the control group $(p=0.046)$ and marginally in the CACR group $(p=0.088)$.

Improvements in cognitive abilities were not associated with symptoms' amelioration. Finally, increase in reasoning abilities was related to the median effective work time in sessions of CACR $(R=0.64, p=0.024)$. Conclusion: At follow-up, enhanced cognitive abilities (reasoning and inhibition), which are necessary for executing higher-order goals or adapting behaviour to the ever-changing environment, were reported in adolescents participants of the CACR. Thus, further studies are needed to confirm and extend these interesting results.

\section{Sébastien Urben', Sandrine Pihet ${ }^{1}$, Laure Jaugey ${ }^{2}$, Olivier Halfon ${ }^{3}$, Laurent Holzer ${ }^{2}$}

${ }^{1}$ Research Unit, University Service of Child and Adolescent Psychiatry, Lausanne, Switzerland; ${ }^{2}$ Day Care Unit for Adolescents (DCUA), University Service of Child and Adolescent Psychiatry, Lausanne, Switzerland; and ${ }^{3}$ University Service of Child and Adolescent Psychiatry, Lausanne, Switzerland

Keywords: adolescents; cognitive remediation; neuropsychology; psychoses

Sébastien Urben, Research Unit, University Service of Child and Adolescent Psychiatry, Route du Bugnon 25A, 1011 Lausanne, Switzerland. Tel: +41 21 3147493;

Fax: +41 21 3147481;

E-mail: Sebastien.Urben@chuv.ch

Accepted for publication January 13, 2012

\section{Significant outcomes}

- This study presented a 6-month follow-up of a randomised single-blind controlled trial assessing a computer-assisted cognitive remediation (CACR) in adolescents with psychotic disorders or at high risk for psychosis.

- Short-term improvements in inhibition and reasoning abilities, independent of amelioration of symptoms, were observed.

- Longer duration of CACR session was reported to be more effective in improving reasoning abilities, raising questions about the dose-effect response of such treatment.

\section{Limitations}

- The small number of participants who completed the 6-month follow-up may limit the generalisation of the results.

- Differential outcomes between psychotic and at high risk for psychosis could not be assessed. 
- Due to the absence of a 'treatment as usual' control group, the effects of the setting (computer use in individual sessions) cannot be disentangled from those of the interventions offered in the day-care centre.

\section{Introduction}

Impairments in a wide range of cognitive abilities have been consistently reported in individuals with psychotic disorders or schizophrenia (1-5). In addition, studies have supported that adolescent illness is associated with a more severe form of schizophrenia and that the length of illness before treatment is negatively related with long-term outcome (6). Specifically, cognitive disabilities in adolescents with psychotic disorders were found to be more severe than in adults, contributing to less independence, poorer educational achievement, vocational failure and difficult social relationships later in adult life $(7,8)$. Furthermore, these cognitive impairments were showed to be present before symptoms onset in individuals who later developed schizophrenia (9) indicating the importance of studying adolescents at risk for psychosis as well.

Although pharmacological treatment has been shown to be effective in reducing psychotic symptoms, particularly positive ones, cognitive impairments have mostly been resistant to such treatments (10). Consequently, an important target of research effort regarding psychotic disorders is the development of effective methods for improving cognitive abilities $(11,12)$. Several reviews of studies, mainly in adult with psychotic disorders (or schizophrenia), have indicated cognitive remediation therapy (CRT) to be effective in reducing cognitive deficits (13-18) with long-term benefits $(19,20)$. Nevertheless, very few studies examined the effect of CRT, and follow-up outcomes, in adolescents with psychotic disorders or at high risk. Only two studies have focused on young individuals (mean age under 20). The first study (21) conducted a 30-h CRT (in addition to the usual psychoeducational treatment program) in adolescents with psychotic disorders (aged from 12 to 18). Although the results indicated improvements in cognitive, clinical and psychosocial domains, there were no gains in favour of the cognitive remediation group compared to the control group and there were no clear benefits at 1-year followup (22). Another randomised controlled trial that assessed the effectiveness of CRT (compared to treatment as usual) in young patients with schizophrenia (aged from 14 to 22) found executive function (cognitive flexibility) and working memory improvements at 6-month follow-up in favour of the CRT group, compared to the control group $(8,23)$.
A specific form of CRT which seems to be of particular interest for improving cognitive abilities in adolescents is the CACR, which provides a structured, flexible and standardised training with clear, accurate and immediate feedback (24). Despite the fact that a meta-analysis did not report higher effect size for CACR compared to paper-and-pencil CRT (16), many arguments could be offered in favour of CACR. First, in adolescents, the use of computer technology is an everyday reality associated with self-perceived competence which is likely to enhance engagement in this form of cognitive remediation (25). Furthermore, computer activities are considered to improve opportunities for acquiring new compensatory strategies, which represent an important component of CRT (26). In addition, prolonged multimedia stimulation is believed to favour neural plasticity (27). In adult patients, CACR programs have been shown to improve cognitive deficits $(28-32)$ as well as psychiatric status $(28,31)$.

However, in adolescents with psychosis or at high risk for psychosis, only one study, conducted by our research group, has assessed CACR outcomes [compared to playing with computer games (CGs)]. The complete analysis of the assessment after the intervention was reported in details elsewhere (33) and may be summarised as follows: improvements in cognition, clinical symptoms and functional outcomes in both groups, but a specific amelioration in visuospatial abilities for the CACR group. Regrettably, the randomised controlled trial was designed in such a way that the assessment after the program and at follow-up was not strictly identical, making it impossible to assess the stability of these improvements. Nevertheless, no study has assessed short-term benefits (i.e. 4 months after the completion of the program) of a CACR in adolescents with psychotic disorders or at high risk for psychosis.

In this context, this study aims to assess short-term outcomes of a CACR program on cognitive abilities and clinical status in adolescents with psychotic disorders or at high risk. The outcomes are compared to a control program involving similar computer use in an individualised setting.

\section{Methods}

Participants

Participants were outpatients, recruited in the Day Care Unit for Adolescents in Lausanne. Informed 
consent was obtained from participants and their guardians. To be included in the study participants had to have (a) a diagnosis of psychotic disorder according to the Diagnostic and Statistical Manual, fourth edition (34), using the French version of Diagnostic Interview for Genetic Studies $(35,36)$ or a diagnosis of high risk of psychosis using the Structured Interview for Prodromal Symptoms and the Scale of Prodromal Symptoms (37) and (b) a score below the 10th percentile in at least one of five domains of the Repeatable Battery for the Assessment of Neuropsychological Status (38). Although arbitrary, the 10th percentile was chosen based on findings from our previous study as this threshold best differentiated patients with psychotic disorders from patients with psychotic disorders from patients with other diagnoses (including mood disorder, anxiety disorders and conduct disorders) which were not reported as having cognitive deficits (2). In addition, the exclusion criteria were as follows: (a) mental retardation (IQ < 70); (b) known neurological disease or developmental disability; (c) severe visual or motor disorder that is incompatible with computer use; (d) transient exclusion criteria: an acute clinical state that could disrupt the CACR training or a planned absence for more than 2 weeks during period of intervention.

Thus, 32 adolescents (psychotic, $N=21$; at high risk for psychosis, $N=11$ ) were considered in the intention-to-treat population. Eighteen were randomised to the treatment condition (CACR) and 14 to the control (CG). Twenty-two participants were assessed at 6-month follow-up (12 in CACR group and 10 in the CG). IQ estimation was obtained with the French version of the National Adult Reading Test (NART) (39). The majority of participants were treated with antipsychotic medication. There were no differences between groups in IQ, proportion of male/female, proportion of psychotic/at risk for psychosis and number of medicated adolescents and number of years of completed education. The Table 1 reports the demographic, clinical characteristics and the statistic tests.

\section{Research design}

The study was approved by the local ethics committee for human research. The design of this study was a single blinded 8-week trial of CACR treatment which was compared to CG. After a baseline assessment and verifying inclusion criteria, participants were randomly assigned to treatment or control group. The psychologists who conducted the neuropsychological assessment were blind to the participant's diagnosis and assignment group and the research psychologists conducting the cognitive
Table 1. Demographic and clinical characteristics of the participants assessed at follow-up

\begin{tabular}{|c|c|c|c|c|c|}
\hline & \multicolumn{2}{|c|}{$\begin{array}{l}\text { CACR group } \\
(N=12)\end{array}$} & \multicolumn{2}{|c|}{$\begin{array}{l}\text { Control group } \\
\qquad(N=10)\end{array}$} & \multirow[b]{2}{*}{$p^{\dagger}$} \\
\hline & Mean* & SD & Mean & SD & \\
\hline Psychotic/at risk for psychosis & $10 / 2$ & - & $6 / 4$ & - & 0.221 \\
\hline Male/female & $7 / 5$ & - & $7 / 3$ & - & 0.675 \\
\hline Age & 15.17 & 1.27 & 16.00 & 1.25 & 0.103 \\
\hline Years completed education & 7.75 & 0.87 & 8.60 & 1.27 & 0.112 \\
\hline IO & 95.05 & 6.48 & 97.80 & 5.90 & 0.247 \\
\hline Medication (\%) at follow-up & 75.0 & - & 70.0 & - & 0.552 \\
\hline
\end{tabular}

* Means were reported for a more usual presentation of the data and because the median (on which the nonparametric test were based) did not greatly differ from these means.

${ }^{\dagger}$ Chi-squared test or Mann-Whitney as appropriate.

training were also blind to the participant's diagnosis. Finally, data analyses were computed by an independent researcher.

\section{Computer-assisted cognitive remediation}

The CACR program was based on the Captain's Log software (40). This program allows training in attention, concentration, memory, visuospatial, visuomotor and conceptualisation with increasing difficulty. Participants were administered two 45-min individual sessions per week over 8 weeks (16 individual sessions). Thirty-minute sessions of training have been shown as effective in improving the cognitive functioning of adults with psychotic disorders (28).

\section{Computer game}

A set of CGs $(N=13$; essentially action CGs that require attention and visuomotor skills) was offered to patients assigned to the control group. The CG program differed from the CACR program only in content (CG instead of Captain's Log software) while the setting was the same (location, computer duration, frequency and trainer). The same psychologist accompanied the patient during all the sessions. The CGs were chosen by the participants and the research psychologist provided encouragement and positive feedback.

\section{Outcome measures}

Cognitive measures. A comprehensive test battery (two different forms of each test were used when it was possible) was administered by a trained neuropsychologist at baseline and 6 months after completing the intervention program (either CACR or CGs). The assessed cognitive functions (i.e. processing speed, memory abilities and executive functions) 
were chosen according to previous studies which reported alteration of these abilities $(1,4,5)$.

Processing speed. The symbol coding test (WAISIII: $>16$ years old/WISC-IV: $<16$ years old) $(41,42)$ was used to assess processing speed abilities. In this test, the participant had to decide if any of two targets symbols were present in a set of five symbols. The main measure was the number of symbols correctly recognised in $120 \mathrm{~s}$.

Working memory. In the letter-number sequencing (WAIS-III: $>16$ years old/WISC-IV: $<16$ years old) $(41,42)$, letters and numbers were presented to the participants who had to recall it in the correct order (alphabetic and increasing number). The measure is the largest span correctly recalled. In the verbal and visuospatial digit span (WMS-III: $>16$ years old/CMS: <16 years old) (43), the participant was presented with clusters of random number sequences of increasing length. They were asked to tell the numbers in forward and backward order, or show the correct sequence of blocks (visuospatial span). The measure was the number of correctly repeated items.

Long-term memory. The Hopkins Verbal Learning Test-Revised (44) was used to assess verbal episodic memory. This test consisted of a list of 12 nouns (targets) with four words drawn from each of three semantic categories. The test was administered by reading the words aloud, then asking the individual to verbally repeat the list of words (immediately and after a delay) and to identify the words from the list from a verbal presentation (including both the target words and the distractors). The measure was the number of words correctly recalled.

The Brief Visuospatial Memory Test-Revised (45) was used to assess visuospatial episodic memory. Patients were presented with six geometric figures printed in a $2 \times 3$ array on separate pages. In the three learning trials, the participant viewed the stimulus for $10 \mathrm{~s}$ and was then asked to draw as many of the figures as possible in their correct location on a page. After a 25-min delay which consisted of primarily verbal activities, the task was repeated. The measures were the number of correct responses recalled.

Executive functions. The Color Stroop task (46) was used to assess one aspect of executive functioning, known as inhibition abilities. In this test, the participant had to suppress the highly overlearned response of word reading in favour of the less automatic process of colour naming. The measure was the number of words processed in $120 \mathrm{~s}$.
The verbal fluency task assessed initiation abilities which are also part of the executive function abilities. In this task, the participant were asked to generate as many words as they could of one category (animals), in a limited time (47). The measure was the number of correct words expressed in $60 \mathrm{~s}$.

Reasoning and planning abilities. The block design test (WAIS-III/WISC-IV) $(41,42)$ assessed reasoning abilities. In this test, participants had to reproduce abstract visuospatial patterns which increase in difficulty. The measure is the number of patterns correctly reproduced.

The Tower of London (48) was administered to the participant in order to assess planning abilities. This test consisted of moving coloured balls within a limited number of moves in order to achieve a given goal configuration. Levels of difficulty depend on the number and complexity of subgoals required. The measure was the total correct score.

For all cognitive tests, standard scores were computed in order to control for age differences and to have more clinical significance. Thus, higher scores reflected better performances.

Symptoms assessment. The global clinical efficacy of the program was assessed with the Clinical Global Impression-Severity of illness scale and Clinical Global Improvement (CGI) (49) which is commonly used as a primary outcome measure in pharmacological studies. The patient's therapist who was blind to his/her group attribution filled the CGI scoring from 0 to 7 .

\section{Data analyses}

The data's distributions were explored using Kolmogorov-Smirnov tests. Results revealed that $45 \%$ of the variables did not have a Gaussian distribution. Thus, in order to compute reliable tests, we computed nonparametric analyses. First, the treatment and the control groups were compared with Mann-Whitney test at baseline in order to ensure comparable performances on the cognitive measures and clinical outcome. Then, using Wilcoxon signed rank tests, performances on cognitive tests and clinical outcome at baseline were compared to the measures at 6-month follow-up, separately for each group. The between-group differences were then assessed at follow-up with Mann-Whitney test. After that, significant changes in cognitive measures were correlated with Spearman's Rho in order to see whether improvement in cognitive measure was related to changes in the severity of the symptom. Correlation analyses were also conducted between 
Urben et al.

Table 2. Baseline and follow-up results for completer participants

\begin{tabular}{|c|c|c|c|c|c|c|}
\hline \multirow[b]{2}{*}{ Variable } & \multirow[b]{2}{*}{ Group } & \multicolumn{2}{|l|}{ Baseline } & \multicolumn{2}{|l|}{ Follow-up } & \multirow[b]{2}{*}{$p^{\S}$} \\
\hline & & Median (SD) & $N$ & Median (SD) & N & \\
\hline \multicolumn{7}{|l|}{ Processing speed } \\
\hline \multirow[t]{2}{*}{ Symbol code } & CACR & $6.50(3.70)$ & 12 & $9.50(3.61)$ & 12 & 0.195 \\
\hline & Control & $9.00(4.47)$ & 10 & $10.00(3.01)$ & 10 & 0.906 \\
\hline \multicolumn{7}{|l|}{ Working memory } \\
\hline \multirow[t]{2}{*}{ Letter-number sequence } & CACR & 7.00 (3.09) & 12 & $8.00(3.75)$ & 12 & 0.385 \\
\hline & Control & $7.50(1.77)$ & 10 & $8.00(2.78)$ & 10 & 0.888 \\
\hline \multirow[t]{2}{*}{ Verbal span } & CACR & $7.50(5.01)$ & 12 & $6.50(4.31)$ & 12 & 0.918 \\
\hline & Control & $8.50(2.80)$ & 10 & $8.50(2.26)$ & 10 & 0.569 \\
\hline \multirow[t]{2}{*}{ Visuospatial span } & CACR & $11.00(3.85)$ & 12 & $10.00(3.58)$ & 12 & 0.325 \\
\hline & Control & $10.00(3.23)$ & 10 & $9.00(3.25)$ & 10 & 0.352 \\
\hline \multicolumn{7}{|l|}{ Episodic memory } \\
\hline \multirow[t]{2}{*}{ Verbal memory } & CACR & $35.00(13.06)$ & 12 & 38.50 (17.08) & 12 & 0.720 \\
\hline & Control & $28.00(15.59)$ & 10 & $38.50(18.27)$ & 10 & 0.593 \\
\hline \multirow[t]{2}{*}{ Visuospatial memory } & CACR & $52.00(15.19)$ & 12 & 44.00 (16.58) & 12 & 0.271 \\
\hline & Control & $59.00(12.20)$ & 10 & $59.00(14.01)$ & 10 & 0.786 \\
\hline \multicolumn{7}{|l|}{ Executive function } \\
\hline \multirow[t]{2}{*}{ Stroop } & CACR & $3.50(1.56)$ & 12 & $5.00(2.10)$ & 12 & $0.040^{\dagger}$ \\
\hline & Control & $5.00(1.90)$ & 10 & $5.00(1.65)$ & 10 & 0.915 \\
\hline \multirow[t]{2}{*}{ Fluency } & CACR & $5.00(0.89)$ & 12 & $5.00(1.14)$ & 12 & 0.705 \\
\hline & Control & $5.00(0.48)$ & 10 & $5.00(0.48)$ & 10 & 1.000 \\
\hline \multicolumn{7}{|l|}{ Reasoning and planning } \\
\hline \multirow[t]{2}{*}{ Block design } & CACR & $8.00(3.93)$ & 12 & $8.50(3.18)$ & 12 & $0.005^{\ddagger}$ \\
\hline & Control & $8.50(4.70)$ & 10 & $8.50(3.67)$ & 10 & 0.551 \\
\hline \multirow[t]{2}{*}{ ToL } & CACR & 100.00 (17.29) & 121 & $103.00(22.10)$ & 12 & 0.778 \\
\hline & Control & $100.00(13.75)$ & & $113.00(18.35)$ & 10 & 0.312 \\
\hline \multicolumn{7}{|l|}{ Symptoms } \\
\hline \multirow[t]{2}{*}{ CGI } & CACR & $5.00(0.75)$ & 12 & $5.00(1.24)$ & 12 & $0.088^{*}$ \\
\hline & Control & $4.00(0.84)$ & 10 & $3.50(1.43)$ & 10 & $0.046^{\dagger}$ \\
\hline
\end{tabular}

${ }^{*} p<.10 ;{ }^{\dagger} p<.05 ;{ }^{\ddagger} p<.01$

${ }^{\S}$ Wilcoxon signed rank test.

the median effective work time in CACR program sessions and the significant changes in cognitive abilities. Finally, completers and non-completers (individuals who did not finish the study) were compared at baseline with Mann-Whitney test.

\section{Results}

Table 2 summarises the performances of each group at baseline and follow-up and the $p$-value of the statistical tests.

At baseline, performances in the fluency task were higher in the control group than in the CACR group $(U=35.00, p=0.024)$. In addition, the severity of symptoms was higher in the CACR group $(U=$ $27.50, p=0.041$ ).

Wilcoxon signed rank tests revealed significant differences between baseline and follow-up, on executive function (Stroop test, $p=0.040, \eta_{\mathrm{p}}{ }^{2}=0.348$ ) and reasoning abilities (block design test, $p=0.005$, $\left.\eta_{\mathrm{p}}^{2}=0.621\right)$ with better performances at follow-up only in the CACR group. In addition, the severity of the symptoms diminished (only marginally significant, $p=0.088, \eta_{p}{ }^{2}=0.273$ ) in the CACR

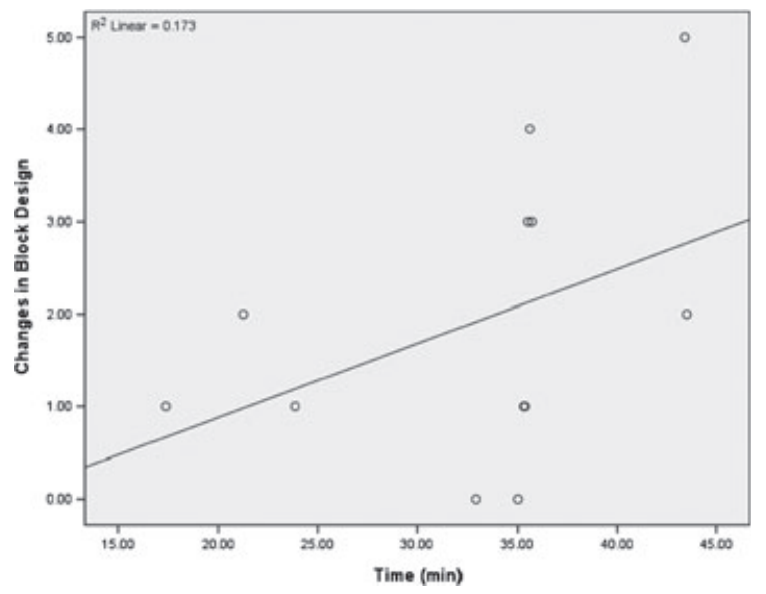

Fig. 1. Relationships between improvement in reasoning abilities (changes in block design test: follow-up - baseline) and median effective work time in sessions.

group as well as in the control group ( $p=0.046$, $\left.\eta_{\mathrm{p}}{ }^{2}=0.400\right)$ between baseline and follow-up.

At follow-up, no significant between-group differences were found on the cognitive measures as well as on the clinical outcome.

No significant correlations were observed between changes in severity of symptoms and changes in the cognitive measures that significantly improved. Furthermore, the median effective work time in sessions of CACR was positively correlated to changes in the block design test ( $R=0.64, p=0.024$, see Fig. 1 ). Significant changes were not related to demographic variables.

Significant differences were found at baseline between participants who withdrew from the study (non-completers) and those who completed the follow-up (completers) on the symbol code $(U=$ 42.50; $p=0.036$; non-completers: median $=11.50$, $\mathrm{SD}=4.73$; completers: median $=8.00, \mathrm{SD}=4.13$ ) and in the block design test $(U=37.50 ; p=0.027$; non-completers: median $=12.50, \mathrm{SD}=3.23$; completers: median $=8.00, \mathrm{SD}=4.35$ ) with the noncompleters showing better performances. However, no differences were found regarding the severity of the symptoms $(p=0.657)$ or group membership (CACR or control, $p=0.959$ ).

\section{Discussion}

This study aimed to explore the long-term outcomes of a CACR in adolescents with psychotic disorders or at high risk for psychosis. At baseline and 6 months after baseline of a CACR or CG program, adolescents were assessed with a comprehensive cognitive battery and the severity of their symptoms was reported by their therapist. Results revealed a specific improvement, only in the CACR group, in executive 
function (inhibition abilities) and reasoning abilities, which represent high-order cognitive capacities. Furthermore, the effective duration of the CACR was related to the magnitude of improvement in reasoning abilities. Symptoms diminished in both groups, but this improvement was independent of the cognitive amelioration. This suggests that CACR has specific effects on some of the investigated cognitive capacities, while the setting (computer use in individual sessions) and/or the treatment program provided in the day-care centre have general effects on symptoms. Due to the absence of a 'treatment as usual' control group, the effects of the setting cannot be disentangled from those of the 'care as usual' interventions. Finally, at baseline, non-completers showed better performances than completers in processing speed and in reasoning ability, and similar performances in other tests, suggesting that our positive findings do not result from a selection bias.

The observed improvement in high-order cognitive abilities is consistent with previous randomised control trials in adolescents with schizophrenia $(8,23)$ which found a long-term improvement in executive function (cognitive flexibility) and working memory. When compared to studies with adults, these results are consistent with a study reporting persistent benefit (4 weeks after discharge) in problem-solving abilities (20). Similarly, another study (19) that proposed a 6-month cognitive remediation focused on working memory abilities, showed a sustained (6 months after the end of the cognitive remediation program) improvement in these abilities. In contrast, another trial in adolescents with psychotic disorders did not find specific improvements for their treatment group $(21,22)$ compared to psychoeducational training (i.e. traditional treatment in their centre) and did not showed long-term benefits. Indeed, after controlling for IQ differences, no clear differences were reported except for one test for which the participants received direct training (22).

The long-term amelioration in abilities such as inhibition and reasoning may allow the individual to better adapt to a changing environment. Indeed, executive functions (more specifically inhibition) are required in order to pursue higher-order goals (50); inhibiting an overlearned response in favour of a non-dominant one represents a necessary step in order to engage in new activities (51). Furthermore, better reasoning abilities could be related to more appropriate decision making. Indeed, it has been shown (52), in healthy participants aged from 6 to 25 years of age, that increased proportional reasoning ability could explain improvements in decision making as measured by the standard Iowa Gambling Task designed to mimic real-life decision making (53).
Thus, improving the reasoning abilities in adolescents with psychotic disorders or at high risk could help them make more appropriate decisions, which may have important long-term benefits (e.g. in social or educational domain) for their lives.

Although practice effects may have interfered in the assessment of these abilities, they are unlikely to have strongly influenced our results for several reasons. First, the cognitive assessment was done with two different versions of the tests, and the repetition occurred after 6 months, which may strongly limit practice effects. Second, results revealed improvements only in the CACR group and not in the control group. Therefore, practice effect is not likely to have produced these results.

No significant relationship was found between ameliorations in cognitive abilities and in symptoms' severity. This result is consistent with previous research in adults with schizophrenia (7) and in adolescents with psychotic disorders $(21,22)$ indicating that improvements in these domains follow different time-course.

A possible limitation is the small number of participants who completed the study, which may have restricted the power of the statistical analyses. However, this population is very difficult to recruit and to assess several times. Moreover, the number of participants in this study did not differ markedly from that of the previous study assessing long-term outcomes of cognitive remediation in a similar population ( $N=31$ in the study of Wykes et al., $N=25$ in the study of Ueland and Rund), underscoring the relevance of this study. Given the preliminary nature of these encouraging results, future research on larger samples is needed to confirm the reported improvements.

Although the heterogeneity of the studied population could be viewed as a possible limitation, cognitive deficits has also been shown in the prodromal phase of the pathology (9), emphasising the need to develop effective interventions for those at high risk for psychosis, whose adolescence process might be impaired by their cognitive deficits. However, studies with large sample size are needed to determine whether the CACR is similarly efficient for adolescents with psychosis and for those at high risk and whether CACR would prevent conversion to psychosis.

The strengths of this study are its randomised single-blind design and its focus on the shortterm outcomes of a CACR program, representing an important topic which has received very little attention. Indeed, it is of particular importance to develop more appropriate treatments targeting cognitive enhancement for adolescents with psychotic 
disorders or at high risk for psychosis, given that they lie at a crucial period of development.

In conclusion, this trial reported long-term improvements in high-order abilities specifically related to an 8-week CACR program. These improvements were not related to symptoms amelioration. Thus, the CACR seems to be a useful tool for the improvement of cognitive abilities, showing promising long-term benefits. Further studies with larger sample are necessary to confirm the improvement found in specific cognitive abilities.

\section{Acknowledgements}

This research was supported by a grant from the Swiss National Science Foundation (no. 32003B_112160). The authors wish to express their gratitude to Mrs Laure Perraudin, Aurélie Aeberhard, Sonja Suter, Virginie Van Craenenbroek, Muriel Hafil, Veronica Pellanda, Raffaella Torrisi and Sandra Barcellona for their involvement in the project and conducting the interventions, and to the patients for their participation in the study. The authors declare no conflict of interest.

\section{References}

1. Asarnow RF, Asamen J, Granholm E, Sherman T, WatKIns JM, WILliams ME. Cognitive/neuropsychological studies of children with a schizophrenic disorder. Schizophr Bull 1994;20:647-669.

2. Holzer L, Chinet L, Jaugey L et al. Detection of cognitive impairment with the Repeatable Battery for the Assessment of Neuropsychological Status (RBANS) in adolescents with psychotic symptomatology. Schizophr Res 2007;95:48-53.

3. Kenny JT, Friedman L, Findling RL et al. Cognitive impairment in adolescents with schizophrenia. Am J Psychiatry 1997;154:1613-1615.

4. Kumra S, Wiggs E, Bedwell J et al. Neuropsychological deficits in pediatric patients with childhood-onset schizophrenia and psychotic disorder not otherwise specified. Schizophr Res 2000;42:135-144.

5. Rhinewine JP, Lencz T, Thaden EP et al. Neurocognitive profile in adolescents with early-onset schizophrenia: clinical correlates. Biol Psychiatry 2005;58:705-712.

6. Schulz SC, Findling RL, Friedman L, Kenny JT, WISE AL. Treatment and outcomes in adolescents with schizophrenia. J Clin Psychiatry 1998;59(Suppl. 1):50-54.

7. Bark N, Revheim N, HuQ F, Khalderov V, Ganz ZW, Medalia A. The impact of cognitive remediation on psychiatric symptoms of schizophrenia. Schizophr Res 2003;63:229-235.

8. Wykes T, Newton E, Landau S, Rice C, ThompSON N, Frangou S. Cognitive remediation therapy (CRT) for young early onset patients with schizophrenia: an exploratory randomized controlled trial. Schizophr Res 2007;94:221-230.

9. Fuller R, Nopoulos P, Arndt S, O’Leary D, Ho BC, ANDREASEN NC. Longitudinal assessment of premorbid cognitive functioning in patients with schizophrenia through examination of standardized scholastic test performance. Am J Psychiatry 2002;159:1183-1189.
10. Nieuwenstein MR, Aleman A, de Haan EH. Relationship between symptom dimensions and neurocognitive functioning in schizophrenia: a meta-analysis of WCST and CPT studies. Wisconsin Card Sorting Test. Continuous Performance Test. J Psychiatr Res 2001;35:119-125.

11. Medalia A, Revheim N, Casey M. Remediation of memory disorders in schizophrenia. Psychol Med 2000;30: $1451-1459$.

12. Bhatia T, Agarwal A, SHanh G et al. Adjunctive cognitive remediation for schizophrenia using yoga: an open, non-randomised trial. Acta Neuropsychiatrica (in press).

13. Krabbendam L, Aleman A. Cognitive rehabilitation in schizophrenia: a quantitative analysis of controlled studies. Psychopharmacology (Berl) 2003;169:376-382.

14. Kurtz MM, Moberg PJ, Gur RC, Gur RE. Approaches to cognitive remediation of neuropsychological deficits in schizophrenia: a review and meta-analysis. Neuropsychol Rev 2001;11:197-210.

15. Pilling S, Bebbington P, Kuipers E et al. Psychological treatments in schizophrenia: II. Meta-analyses of randomized controlled trials of social skills training and cognitive remediation. Psychol Med 2002;32:783-791.

16. Twamley EW, Jeste DV, Bellack AS. A review of cognitive training in schizophrenia. Schizophr Bull 2003;29: 359-382.

17. McGurk SR, Twamley EW, Sitzer DI, McHugo GJ, MuESER KT. A meta-analysis of cognitive remediation in schizophrenia. Am J Psychiatry 2007;164:1791-802.

18. Wykes T, Huddy V, Cellard C, McGurk SR, Czobor P. A meta-analysis of cognitive remediation for schizophrenia: methodology and effect sizes. Am J Psychiatry 2011;168:472-485.

19. Bell M, Bryson G, Wexler BE. Cognitive remediation of working memory deficits: durability of training effects in severely impaired and less severely impaired schizophrenia. Acta Psychiatr Scand 2003;108:101-109.

20. Medalia A, Revheim N, Casey M. Remediation of problem-solving skills in schizophrenia: evidence of a persistent effect. Schizophr Res 2002;57:165-171.

21. Ueland T, Rund BR. A controlled randomized treatment study: the effects of a cognitive remediation program on adolescents with early onset psychosis. Acta Psychiatr Scand 2004;109:70-74.

22. Ueland T, Rund BR. Cognitive remediation for adolescents with early onset psychosis: a 1-year follow-up study. Acta Psychiatr Scand 2005;111:193-201.

23. Wykes T, Reeder C, Landau $\mathrm{S}$ et al. Cognitive remediation therapy in schizophrenia: randomised controlled trial. Br J Psychiatry 2007;190:421-427.

24. Medalia A, Aluma M, Tryon W, Merriam AE. Effectiveness of attention training in schizophrenia. Schizophr Bull 1998;24:147-152.

25. Bremer J, RAuCh PK. Children and computers: risks and benefits. J Am Acad Child Adolesc Psychiatry 1998;37:559-560.

26. Kurtz MM, Seltzer JC, Shagan DS, Thime WR, WeXLer BE. Computer-assisted cognitive remediation in schizophrenia: what is the active ingredient? Schizophr Res 2007;89:251-260.

27. Hogarty GE, Flesher S, Ulrich R et al. Cognitive enhancement therapy for schizophrenia: effects of a 2year randomized trial on cognition and behavior. Arch Gen Psychiatry 2004;61:866-876. 
28. Bellucci DM, Glaberman K, Haslam N. Computerassisted cognitive rehabilitation reduces negative symptoms in the severely mentally ill. Schizophr Res 2003;59:225-232.

29. Burda PC, Starkey TW, Dominguez F, Vera V. Computer-assisted cognitive rehabilitation of chronic psy chiatric-inpatients. Comput Human Behav 1994;10:359-368.

30. Fisher M, Holland C, Merzenich MM, Vinogradov S. Using neuroplasticity-based auditory training to improve verbal memory in schizophrenia. Am J Psychiatry 2009;166: 805-811.

31. Medalia A, Aluma M, Tryon W, Merriam AE. Effectiveness of attention training in schizophrenia. Schizophr Bull 1998;24:147-152.

32. Grynszpan O, Perbal S, Pelissolo A et al. Efficacy and specificity of computer-assisted cognitive remediation in schizophrenia: a meta-analytical study. Psychol Med 2011;41:163-173.

33. Holzer L, Passini MC, Pellanda V et al. A randomised controlled trial of the effectiveness of a computer-assisted cognitive remediation (CACR) program in adolescents with psychosis or at high risk of psychosis. Behav Res Ther (submitted).

34. APA. Diagnostic and statistical manual of mental disorders (DSM-IV), 4th edn. Washington (DC): American Psychiatric Association, 1994.

35. Nurnberger Ji, Blehar MC, Kaufmann CA et al. Diagnostic interview for genetic-studies - rationale, unique features, and training. Arch Gen Psychiatry 1994;51:849-859.

36. Preisig M, Fenton BT, Matthey ML, Berney A, FerRERO F. Diagnostic interview for genetic studies (DIGS): inter-rater and test-retest reliability of the French version. Eur Arch Psychiatry Clin Neurosci 1999;249:174-179.

37. Miller TJ, McGlashan TH, Woods SW et al. Symptom assessment in schizophrenic prodromal states. Psychiatr Q 1999;70:273-287.

38. Randolph C, Tierney MC, Mohr E, Chase TN. The Repeatable Battery for the Assessment of Neuropsychological Status (RBANS): preliminary clinical validity. J Clin Exp Neuropsychol 1998;20:310-319.

39. Mackinnon A, Mulligan R. The estimation of premorbid intelligence levels in French speakers. Encephale 2005;31:31-43.

40. Sanford JA, Brown RJ. Captain's log cognitive system. Richmond: Brain Train, Inc., 1988.
41. WeChSLER D. WAIS III: Echelle d'intelligence pour adultes, 3ème. Paris: ECPA, 1997.

42. WeChSLER D. WISC IV: Echelle d'intelligence pour enfants, 4ème. Paris: ECPA, 2005.

43. WeChSLER D. MEM III: Echelle clinique de mémoire de Wechsler, 3ème. Paris: ECPA, 1997.

44. BRAndT J. The Hopkins Verbal Learning Test: development of a new verbal memory test with six equivalent forms. Clin Neuropsychol 2005;5:125-142.

45. Benedict RHB, Schretlen D, Groninger L, DobRASKI M, SHPRITZ B. Revision of the brief visuospatial memory test: studies of normal performance, reliability, and validity. Psychol Assess 1996;8:145-153.

46. STroop JR. Studies of interference in serial verbal reactions. J Exp Psychol 1935;18:643-662.

47. Sauzeon H, Lestage P, Raboutet C, N'Kaoua B, Claverie B. Verbal fluency output in children aged 7-16 as a function of the production criterion: qualitative analysis of clustering, switching processes, and semantic network exploitation. Brain Lang 2004;89:192-202.

48. Krikorian R, BARTOK J, GAY N. Tower of London procedure: a standard method and developmental data. J Clin Exp Neuropsychol 1994;16:840-850.

49. GuY W. ECDEU assessment manual. Rockville (Maryland): US Department of Health, Education and Welfare, 1976.

50. Miyake A, Friedman NP, Emerson MJ, Witzki AH, Howerter A, Wager TD. The unity and diversity of executive functions and their contributions to complex "Frontal Lobe" tasks: a latent variable analysis. Cogn Psychol 2000;41:49-100.

51. LOGAN GD. On the ability to inhibit thought and action: a users' guide to the stop signal paradigm. In: DAGENBACH D, CARR TH, eds. Inhibitory processes in attention, memory, and language. London: Academic Press Inc., 1994: 189-239.

52. Huizenga HM, Crone EA, Jansen BJ. Decision-making in healthy children, adolescents and adults explained by the use of increasingly complex proportional reasoning rules. Dev Sci 2007;10:814-825.

53. Bechara A, Damasio AR, Damasio H, Anderson SW. Insensitivity to future consequences following damage to human prefrontal cortex. Cognition 1994;50:7-15. 